\title{
Steric and Electronic Interactions at GIn154 in ZEITLUPE Induce Reorganization of the LOV Domain Dimer Interface
}

Authors: Ashutosh Pudasaini ${ }^{1 \dagger}$, Robert Green ${ }^{1 \dagger}$, Young Hun Song ${ }^{2}$, Abby Blumenfeld ${ }^{1}$, Nischal Karki $^{1}$, Takato Imaizumi ${ }^{3}$, Brian D. Zoltowski ${ }^{1 *}$

${ }^{1}$ Department of Chemistry, Southern Methodist University, Dallas, TX 75275, USA

Center for Drug Discovery, Design and Delivery, Southern Methodist University

${ }^{2}$ Department of Agricultural Biotechnology, Seoul National University, Seoul, 08826, Korea

${ }^{3}$ Department of Biology, University of Washington, Seattle, WA, 98195-1800, USA

${ }^{*}$ corresponding author:

Brian D. Zoltowski
Associate Professor, Department of Chemistry, Southern Methodist University
Dallas, TX 75275-0314
t. 214-768-2640
f. 214-768-4089
e. bzoltowski@smu.edu

$\dagger$ Have contributed equally to this work. 
Supporting information:

Supporting Figure S1: Alternative assemblies in the crystal lattice and GIn154 orientations

Supporting Figure S2: SEC-SAXS data and analysis

Supporting Figure S3: Comparison of LOV structures containing a Gly at position 46

A
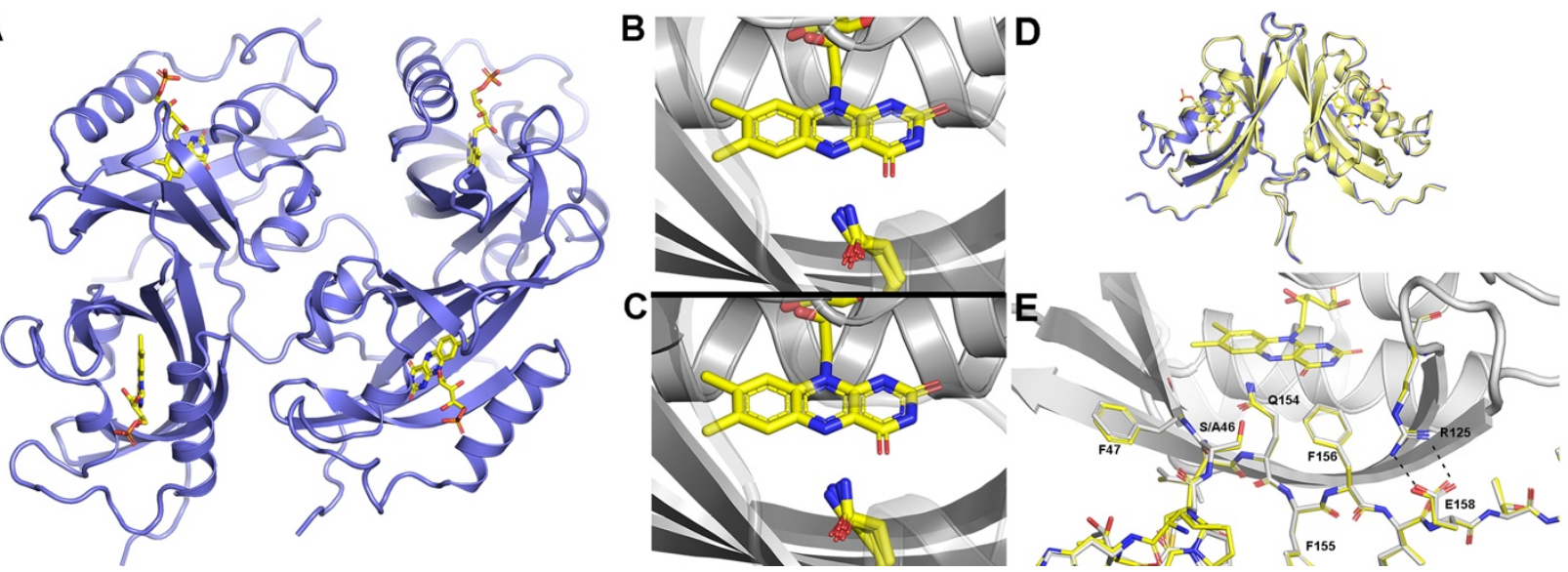

Fig. S1: G46S and G46A Structural Configurations. A) A tetrameric assembly present in the crystal structures. Dimeric, tetrameric, and the heptameric rings can be found within different selections of the asymmetric unit. Only the dimeric assembly has been observed in solution studies. B, C) All seven GIn154 orientations within the G46S (B) and G46A (C) crystal lattice. In all molecules the GIn side chain adopts a configuration that is partially buried to allow $\mathrm{H}$-bonding interactions at $\mathrm{N} 5$ and $\mathrm{O} 4$ of the flavin isoalloxazine ring. D) G46S (yellow) and G46A (blue) generate equivalent parallel dimers. E) Comparison of G46S (yellow) and G46A (grey) signaling elements. In both proteins equivalent structures are observed for the CGF and QFF motifs, and both proteins demonstrate formation of a salt-bridge between Arg125-Glu158. 

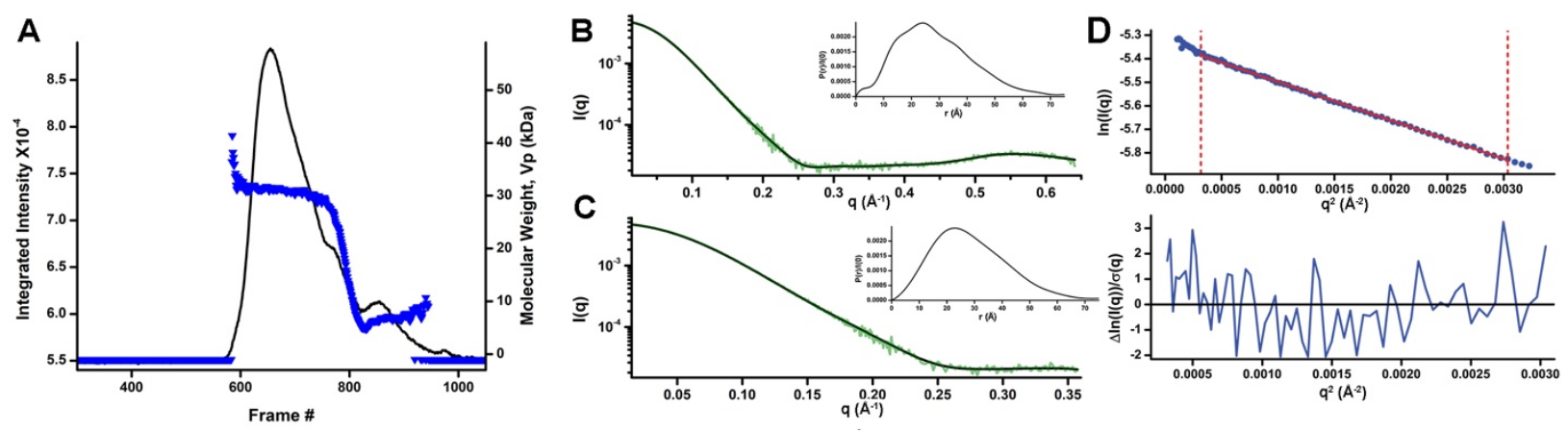

Fig. S2: SEC-SAXS of G46S:G80R ZTL. A) G46S:G80R elutes predominantly as a dimer with a secondary peak due to sample degradation (concentration at loading $=20 \mathrm{mg} / \mathrm{ml}$ ). SAXS profiles were generated using frames 631-696 to capture the primary dimer peak. B) Full scattering profile for G46S:G80R from merged SAXS-WAXS data (olive) and corresponding fit of the pairwise distribution function (black line). Pairwise distribution profiles (inset) were generated using GNOM and a Dmax=77 A. C) Scattering profile truncated to q_max=8/Rg for DAMMIN reconstructions (olive) and corresponding fit of the pairwise distribution function (black line). Pairwise distribution profiles (inset) were generated using GNOM. D) Guinier analysis with residuals results in an $\mathrm{Rg}=22.2 \AA$. Dashed lines represent the region fit for Guinier analysis. SAXS data including reconstructions are available in the SASBDB (SASDJX5). 


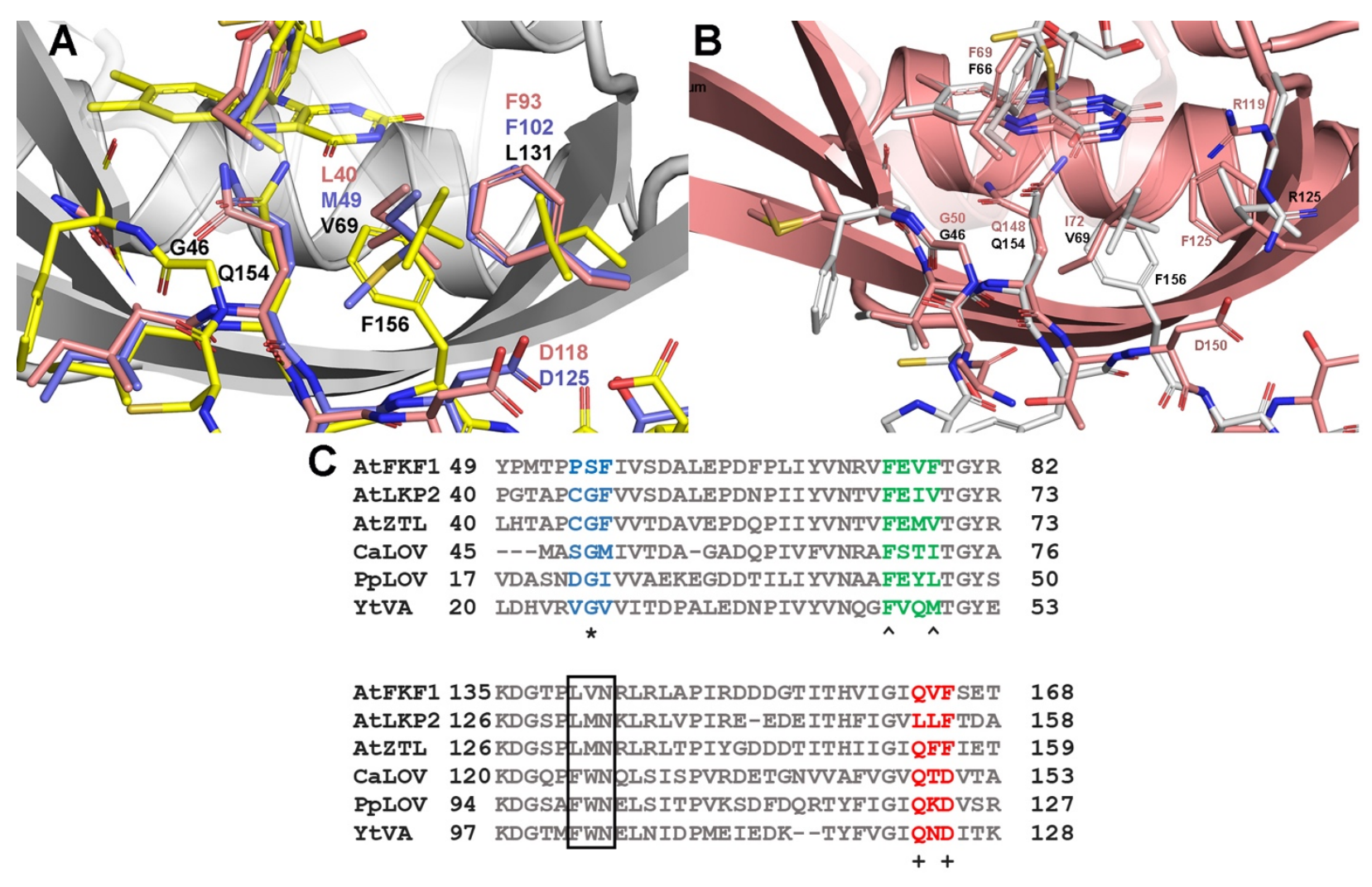

Fig. S3: LOV Structures with Gly residues at position 46. A) Comparisons of YtvA (Blue; PDB: 2PR5), PpLOV (Salmon; PDB: 5J3W), and ZTL (Yellow; PDB: 5SVG) active sites. All proteins contain a Gly at position 46 and have demonstrated alternative conformations of the active site GIn. ZTL is unique in containing Phe156 inserted into the active site pocket. In other LOV proteins this residue is solvent exposed. In YtvA and PpLOV the equivalent residue (D125 and D118 respectively) is involved in light dependent salt-bridge formation. An adjacent site (ZTL L131), contains a Phe residue in most LOV domains, but contains a Leu in ZTL group proteins. B) Comparison of light-state ZTL V48I:G80R (grey; PDB: 5SVW) and CaLOV (Salmon; PDB: 6RHG). Both proteins contain a Gly at position 46, and Ile at position 48, and demonstrate occupancy of both buried and exposed conformations of Gln154 (Gln148 in CaLOV). CaLOV also contains a solvent exposed Asp residue at the position equivalent to Phe156. No light-state structures exist to indicate if it is involved in salt-bridge formation. Black sequence labels represent ZTL and Salmon represents CaLOV. C) Sequence alignment of ZTL group proteins in A. thaliana and LOV proteins with Gly residues at position 46 that have been structurally characterized. The CGF motif (blue) and QFF motif (red) demonstrate divergence of FKF1, which contains a Ser residue at the equivalent position to Gly46 (asterisk). LKP2 tolerates a Leu residue at position 154 (+ sign). ZTL group proteins conserve a Phe residue (Phe156: + sign) within the QFF motif that is solvent exposed and involved in salt-bridge formation in YtvA, and PpLOV. Residues in $\mathrm{C} \alpha$ interact with the QFF motif. These include conserved Phe residues undergoing conformational changes in YtvA and PpLOV (Phe46 and Phe37 respectively: ${ }^{\wedge}$ symbol), and a residue one turn further along the helix (^ symbol) that normally fills the pocket occupied by Phe156 in ZTL. In ZTL, this site contains a smaller residue (Val69), which accommodates Phe156 in the flavin active site. These residues (^ symbol) couple $\mathrm{C} \alpha$ to the LOV C-terminus. The black box highlights a divergent Leu residue in the flavin pocket that is a Phe in most other LOV proteins. 